$7-2-2014$

\title{
Modified active disturbance rejection control for time-delay systems
}

\author{
Shen Zhao \\ Michigan State University, zhaos@frib.msu.edu \\ Zhiqiang Gao \\ Cleveland State University, Z.GAO@csuohio.edu
}

Follow this and additional works at: https://engagedscholarship.csuohio.edu/enece_facpub

How does access to this work benefit you? Let us know!

Publisher's Statement

NOTICE: this is the author's version of a work that was accepted for publication in ISA

Transactions. Changes resulting from the publishing process, such as peer review, editing, corrections, structural formatting, and other quality control mechanisms may not be reflected in this document. Changes may have been made to this work since it was submitted for publication. A definitive version was subsequently published in ISA Transactions, 53, 4, (07-02-2014); 10.1016/j.isatra.2013.09.013

\section{Repository Citation}

Zhao, Shen and Gao, Zhiqiang, "Modified active disturbance rejection control for time-delay systems" (2014). Electrical Engineering \& Computer Science Faculty Publications. 281.

https://engagedscholarship.csuohio.edu/enece_facpub/281

This Article is brought to you for free and open access by the Electrical Engineering \& Computer Science Department at EngagedScholarship@CSU. It has been accepted for inclusion in Electrical Engineering \& Computer Science Faculty Publications by an authorized administrator of EngagedScholarship@CSU. For more information, please contact library.es@csuohio.edu. 


\title{
Modified active disturbance rejection control for time-delay systems
}

\author{
Shen Zhao *, Zhiqiang Gao \\ Center for Advanced Control Technologies, Fenn College of Engineering. Cleveland State University, Cleveland, $\mathrm{OH} 44115$, United States
}

\section{Introduction}

Most industrial processes, e.g. combustion, distillation, waste water treatment, are often treated as either first order plus time delay (FOPTD) systems or second order plus time delay (SOPTD) systems to simplify the controller design. The time delay, also known as dead time, is generally associated with the transportation of the material or energy in the processes [1]. In addition, it may be the result of an approximation of a higher order dynamics with a lower order one, which is not the main focus of this paper.

The control design for time-delay systems is very challenging due to the fact that the time delay introduces additional phase lag, which increases with frequency, to the system, which reduces the stability margin or simply destabilizes it. Hence the achievable closed-loop bandwidth is normally limited to $1 / \tau[2]$, where $\tau$ is the time delay. The well-known Smith predictor [3] has been the main method of choice to deal with such systems, since it can increase the closed-loop bandwidth by removing the time delay from the loop. The prerequisite is an accurate system model is available; otherwise the high bandwidth may cause instability due to model uncertainties. Although the tracking performance is enhanced, the disturbance rejection performance of the original Smith predictor is quite limited. In addition, it cannot deal with time-delay systems which have right half plane poles. To improve its performance numerous efforts have been made to modify the original Smith predictor [4]. In particular, the control of integral

\footnotetext{
* Corresponding author. Presently at: Facility for Rare Isotope Beams and Nationa Superconducting Cyclotron Laboratory. Michigan State University, East Lansing. MI 48824, USA. Tel.: + 1517908 7234; fax: +1 5179087126

E-mail address: zhaos@frib.msu.edu (S. Zhao).
}

processes with time delay seems to attract much attention [5-8]. Zhong et al. wrote a series of four papers on this topic [9-12], proposing a disturbance observer based approach. There are also efforts on finding a unified solution for stable, integral or even unstable time-delay systems $[13,14]$. All of the above Smith predictor based methods, however, start with a fairly good mathematical model of the system.

In the absence of such a model, the active disturbance rejection control (ADRC) [15], which is known for its ability to accommodate both external disturbances and internal uncertainties, seems to be a viable alternative. The essence of ADRC is to treat the whole effect of both external disturbances and internal uncertainties as total disturbance, then estimate it using the extended state observer (ESO) and cancel it out in the control law. The ADRC has been successfully applied to various applications [16-20] and recent theoretical analysis of it can be found in [21-24], but mainly for systems without time delay.

The application of ADRC to time-delay systems has been studied by other researchers $[25,26]$ as well. Several methods was proposed in [25] to deal with time delay in the ADRC design. The first one is to ignore the time delay and design the ADRC for the dynamics without time delay. This leads to limited performance. The second method approximates the time delay with a first order dynamic using the relation $e^{-\tau s} \approx 1 /(\tau S+1)$ and adopts a higher order ADRC design. Other methods try to predict either the system output or the control signal based on the Taylor series, i.e., $\theta(t+\tau) \approx \theta(t)+\dot{\theta}(t) \tau$, when the time delay $\tau$ is small. The ADRC design for a multivariable time-delay system is studied in [26], where the approximation method is adopted. The original nonlinear ADRC designs in $[25,26]$, though provide a relatively satisfactory performance, are rather too complex for practical applications. It is the aim of this paper to 
provide an effective and relatively easy to implement ADRC solution to the prevailing industrial process control applications.

The paper is organized as follows. In Section 2, the regular ADRC design is first introduced followed by the proposed modification to it. In Section 3, simulation and experimental results are provided to demonstrate the effectiveness of the proposed design. Section 4 provides the stability analysis for the closed-loop system applying the proposed design and Section 5 concludes the paper.

\section{Active disturbance rejection control}

\subsection{The regular design}

A simple motion control problem is used to illustrate the regular ADRC design. Consider the following system dynamics.

$m \ddot{y}=F+g(y, \dot{y}, t)+w(t)$

where $m$ is the mass, $y$ is the position, $F$ is the control force, $w$ is the disturbance force, $t$ is the time and $g(y, \dot{y}, t)$ is a nonlinear time-varying function of the position and velocity, which may correspond to nonlinear spring and friction forces. In the context of active disturbance rejection, the original system (1) is reformulated as

$\ddot{y}=b u+f(y, \dot{y}, w, t)$

where $b=1 / m, u=F$ and $f(y, \dot{y}, w, t)=(g(y, \dot{y}, t)+w(t)) / m$ is called the total disturbance [15] which includes not only the external disturbances but also the unknown internal dynamics. Then the state vector of the system is defined as $\mathbf{x}=\left[\begin{array}{lll}x_{1} & x_{2} & x_{3}\end{array}\right]^{T}=$ $\left[\begin{array}{lll}y & \dot{y} & f\end{array}\right]^{T}$, which has three components. Note that for a second order system the state vector is normally defined as $\mathbf{x}=\left[\begin{array}{ll}y & \dot{y}\end{array}\right]^{T}$ with two components. Here $x_{3}=f$, which is called the extended state representing the total disturbance, is augmented to the regular design.

The state space representation of Eq. (2) is

$\dot{\mathbf{x}}=\mathbf{A} \mathbf{x}+b \mathbf{B} u+\mathbf{E} \dot{f}$

$y=\mathbf{C x}$

where

$\mathbf{A}=\left[\begin{array}{lll}0 & 1 & 0 \\ 0 & 0 & 1 \\ 0 & 0 & 0\end{array}\right], \mathbf{B}=\left[\begin{array}{l}0 \\ 1 \\ 0\end{array}\right], \mathbf{E}=\left[\begin{array}{l}0 \\ 0 \\ 1\end{array}\right]$, and $\mathbf{C}=\left[\begin{array}{lll}1 & 0 & 0\end{array}\right]$.

An ESO is designed for system (3) accordingly as

$\dot{\hat{\mathbf{x}}}=\mathbf{A} \hat{\mathbf{x}}+\hat{b} \mathbf{B} u+\mathbf{L}\left(x_{1}-\hat{x}_{1}\right)$

where $\hat{\mathbf{x}}=\left[\begin{array}{lll}\hat{x}_{1} & \hat{x}_{2} & \hat{x}_{3}\end{array}\right]^{T}$ is the observer state vector which provides an estimation of the system state vector $\mathbf{x}, \hat{b}$ is an estimation of $b$, and $\mathbf{L}=\left[\begin{array}{lll}l_{1} & l_{2} & l_{3}\end{array}\right]^{T}$ is the observer gain vector. The controller is designed as

$u=\mathbf{K}(\mathbf{r}-\hat{\mathbf{x}}) / \hat{b}$

where $\mathbf{r}=\left[\begin{array}{lll}r & \dot{r} & \ddot{r}\end{array}\right]^{T}, r$ is the reference signal, and $\mathbf{K}=\left[\begin{array}{lll}k_{1} & k_{2} & 1\end{array}\right]$ is the controller gain vector. In practice, $\dot{r}$ and $\ddot{r}$ are set to zero if they are either not available or unbounded.

According to the parameterization technique proposed in [27], the individual observer gains $l_{i}(i=1,2,3)$ are selected such that all eigenvalues of $\mathbf{A}-\mathbf{L C}$ are placed at $-\omega_{0}$, and they are found to be $l_{1}=3 \omega_{0}, l_{2}=3 \omega_{0}^{2}$ and $l_{3}=\omega_{0}^{3}$ in this case. Similarly, the individual controller gains $k_{i}(i=1,2)$ are selected such that all eigenvalues of matrix $\tilde{\mathbf{A}}_{2 \times 2}$ are placed at $-\omega_{c}$, where $\tilde{\mathbf{A}}_{2 \times 2}$ is defined as

$\left[\begin{array}{cc}\tilde{\mathbf{A}}_{2 \times 2} & 0 \\ 0 & 0\end{array}\right]=\mathbf{A}-\mathbf{B K}$

and they are found to be $k_{1}=\omega_{c}^{2}$ and $k_{2}=2 \omega_{c}$. Above, $\omega_{0}$ and $\omega_{c}$ are referred to as observer and controller bandwidth respectively, and are the tuning parameters of the ADRC design.

\subsection{Modified ADRC design to accommodate time delay}

The modification to the regular ADRC design is straightforward and intuitive. A time delay block is added, as shown in Fig. 1, to delay the control signal before it goes into the extended state observer. Since the system output is already delayed due to the system dynamic, this will synchronize the signals that go into the observer and allow it to provide meaningful estimations of the delayed system states and delayed disturbances.

Remark: This synchronization only removes the time delay from the observer loop, unlike in the Smith predictor where the time delay is removed from the main loop. Hence the closed-loop bandwidth of the modified design is still limited. To improve the tracking performance, however, the feedforward control can be used as an alternative, as it has been shown to be effective in [28].

Compare to the regular ADRC design described in Section 2.1, the proposed ADRC can be implemented by replacing Eq. (4) with the following.

$\dot{\hat{\mathbf{x}}}(t)=\mathbf{A} \hat{\mathbf{x}}(t)+\hat{b} \mathbf{B} u(t-\tau)+\mathbf{L}\left(x_{1}(t)-\hat{x}_{1}(t)\right)$

Though the modification is simple, it enhances the regular ADRC design by increasing achievable observer bandwidth, which is the key for an accurate estimation of the total disturbance. With appropriate tuning, the proposed method also provides a unified solution to a variety of time-delay systems (with stable, critical stable, or unstable poles), as will be demonstrated in Section 3.

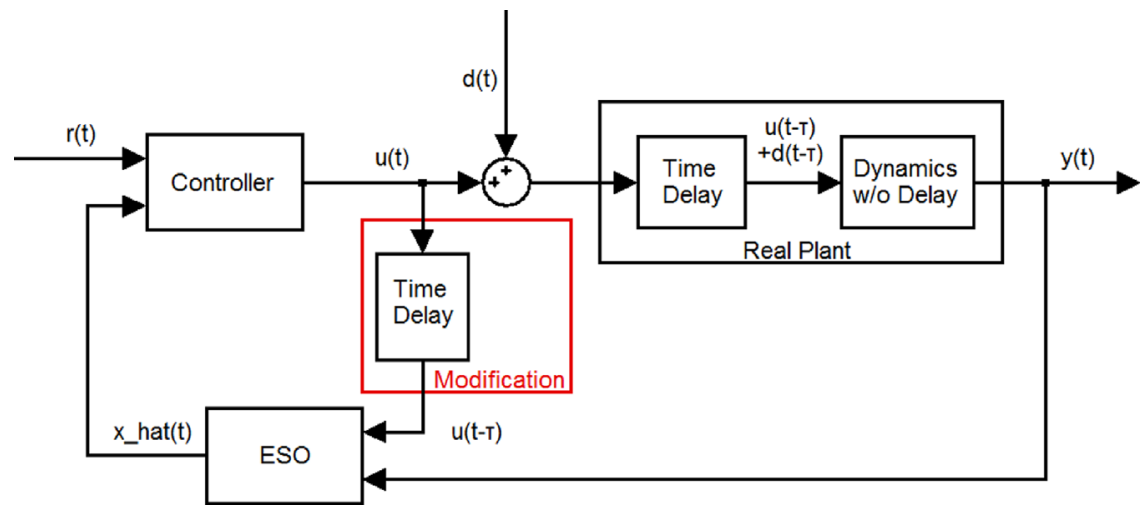

Fig. 1. Modified ADRC for time-delay systems. 


\subsection{Tuning rules}

In practice, the observer bandwidth and the controller bandwidth are selected based on following considerations: (1) the controller bandwidth should be high enough to meet the design specifications; (2) the observer bandwidth should be two to ten times higher than the controller bandwidth; and (3) the observer bandwidth should also be five to ten times less than the sampling rate. Normally the higher the bandwidth, the better the performance; the cost is that the system is more susceptible to noise and has less robustness.

Specifically for time-delay systems, the controller bandwidth can be set to $1 / \tau$ initially as indicated by [2] and adjusted further. If perfect synchronization is achieved, i.e. the time delay added matches exactly the time delay in the real system, the observer bandwidth is only limited by the sampling rate and the acceptable noise level. Therefore it can be set as high as possible, which is especially desired for unstable time-delay system. Since higher observer bandwidth generally gives better states and disturbance estimations. In the case that the information about the time delay is not exactly known, properly lowering the observer bandwidth will ensure the stability, at the cost of poor disturbance rejection.

\section{Simulation and experimental results}

The following FOPTD and SOPTD systems have been widely studied $[1,29]$ and will be used to test the proposed method in this section.

$G_{\text {FOPTD }}(s)=\frac{b}{s+a} e^{-\tau s}$

$G_{S O P T D}(s)=\frac{b}{s^{2}+a_{1} s+a_{0}} e^{-\tau s}$

In most cases $a>0, a_{1}>0$ and $a_{0}>0$, i.e. all the poles are stable. With $a=0$ and $a_{0}=0$, system (7) and (8) become integral processes with time delay, which has caught much attention in the literatures. Furthermore $a<0$, and $a_{1}<0$ or $a_{0}<0$, are the unstable cases, which make the problem even more challenging.

\subsection{Simulation results}

Example 1. The fuel dynamics of a boiler turbine unit is modeled as a FOPTD system in [30] with the following transfer function,

$G_{f u e l}(s)=\frac{Y(s)}{U(s)}=\frac{6.31}{145 s+1} e^{-60 s}=\frac{4.35 \times 10^{-2}}{s+6.90 \times 10^{-3}} e^{-60 s}$

where the input is the fuel rate fed into the furnace and the output is the power generated by burning the fuel.

Three different ADRC designs are compared. The first one is a standard first order ADRC by ignoring the time delay; the second design is a standard second order ADRC approximating the time delay with a first order dynamic; the last is the modified ADRC. The parameters of the three ADRC designs are chosen as for the regular first order ADRC, $\hat{b}=4.35 \times 10^{-2}, \omega_{c}=0.015 \mathrm{rad} / \mathrm{s}$ and $\omega_{0}=0.015 \mathrm{rad} / \mathrm{s}$; for the regular second order ADRC, $\hat{b}=2.18 \times$ $10^{-3}, \omega_{c}=0.02 \mathrm{rad} / \mathrm{s}$ and $\omega_{0}=0.04 \mathrm{rad} / \mathrm{s}$; for the modified first ADRC, $\hat{b}=4.35 \times 10^{-2}, \omega_{c}=0.015 \mathrm{rad} / \mathrm{s}$ and $\omega_{0}=0.15 \mathrm{rad} / \mathrm{s}$. An external disturbance with a magnitude of $3 \mathrm{t} / \mathrm{h}$ is added at $25 \mathrm{~min}$. The simulation results are shown in Fig. 2.

As can be seen from results, the modified ADRC provides the best disturbance rejection (with lowest maximum error and shortest settling time) among the three, owing to the higher observer bandwidth achieved by the synchronization modification. This

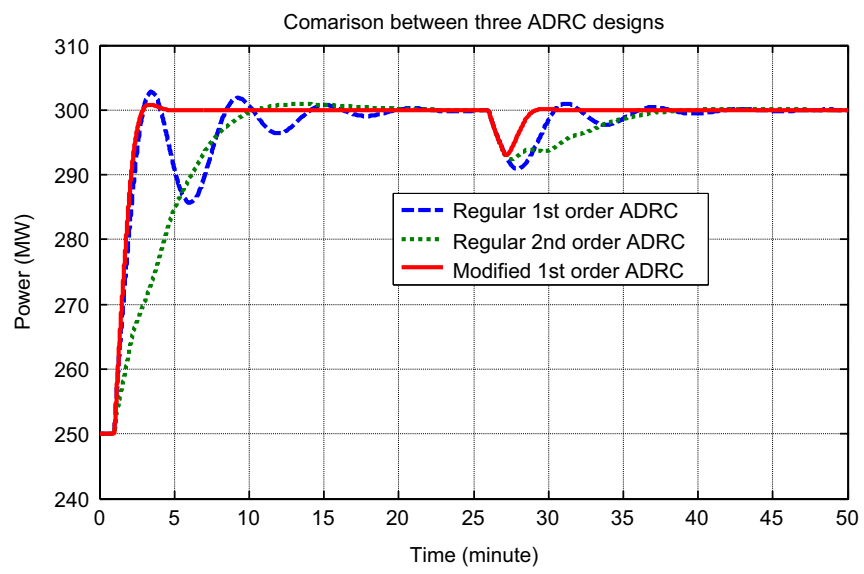

Fig. 2. Simulation results of three ADRC designs for the FOPTD system.

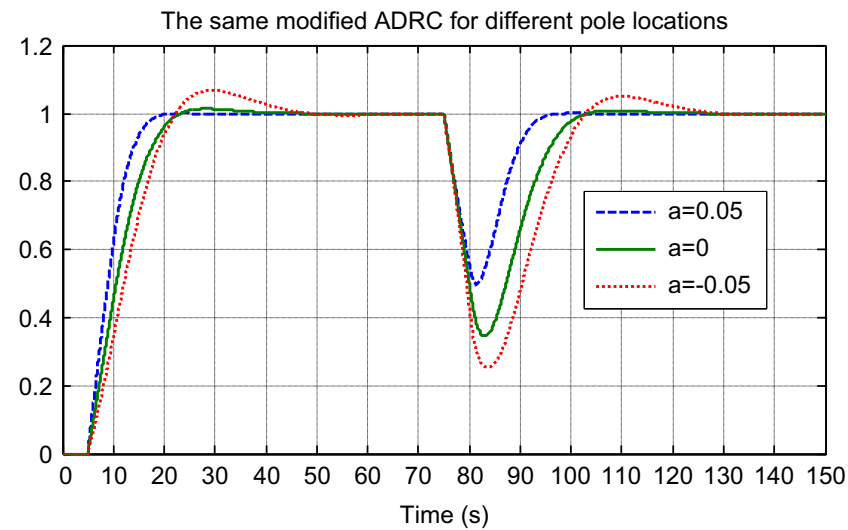

Fig. 3. Simulation results of ADRC accommodating different system dynamics.

demonstrates the advantages of the modified ADRC over the standard ADRC for time-delay systems.

Example 2. In this example, the ability of the proposed ADRC to deal with different types of time-delay systems is tested. The systems studied are all in the same form of Eq. (7) with parameters $\tau=5, b=1$ and $a=0.05,0$ and -0.05 respectively. The design parameter $\hat{b}=b=1$ is the same for all three cases, and the tuning parameters are chosen as: for $a=0.05, \omega_{c}=0.14 \mathrm{rad} / \mathrm{s}$ and $\omega_{0}=$ $1.4 \mathrm{rad} / \mathrm{s}$; for $a=0, \omega_{c}=0.09 \mathrm{rad} / \mathrm{s}$ and $\omega_{0}=0.9 \mathrm{rad} / \mathrm{s}$; for $a=$ $-0.05, \omega_{c}=0.06 \mathrm{rad} / \mathrm{s}$ and $\omega_{0}=1.8 \mathrm{rad} / \mathrm{s}$. An external disturbance with a magnitude of 0.1 is added at $70 \mathrm{~s}$. The simulation results are shown in Fig. 3.

It is demonstrated in this example that the same ADRC design works for time-delay systems with stable, critical stable and unstable poles, providing a unified solution. Notice that, for the $a=0$ case, similar performance as given in [5] is achieved, but the proposed controller is easier to tune. Basically only one tuning parameter needs to be adjusted, whereas in [5], there are three parameters for the filter and one for the controller, all need to be tuned.

Example 3. This example is intended to demonstrate that ADRC works not only for linear time invariant (LTI) systems but also for nonlinear, time-varying systems, with unknown dynamics. Consider the following system.

$\ddot{y}(t)+y(t) \dot{y}(t)+0.02 \sin (0.6 t) y(t)=u(t-0.5)$

A modified second order ADRC is designed for system (10) with the following parameters: $\hat{b}=1, \omega_{c}=1 \mathrm{rad} / \mathrm{s}$ and $\omega_{0}=10 \mathrm{rad} / \mathrm{s}$, in the absence of any knowledge of the model other than its order 
and an estimation of $b$. An external disturbance with a magnitude of 1 is added at $30 \mathrm{~s}$. The simulation results are shown in Fig. 4.

The system settles and recovers from disturbance in $5 \mathrm{~s}$. The slight ripples in the response are caused by the time-varying effect of the system. Note that the modified ADRC can deal with system uncertainties even for time-delay systems.

\subsection{Experimental results}

The distillation column benchmark problem [31] is studied here. The system has two inputs and two outputs, and the system dynamics is represented by the following transfer function matrix.

$$
\left[\begin{array}{l}
Y_{1}(s) \\
Y_{2}(s)
\end{array}\right]=\left[\begin{array}{ll}
P_{11}(s) & P_{12}(s) \\
P_{21}(s) & P_{22}(s)
\end{array}\right]\left[\begin{array}{l}
U_{1}(s) \\
U_{2}(s)
\end{array}\right]
$$

where $P_{11}(s)=12.8 e^{-1 s} / 16.7 s+1, \quad P_{12}(s)=-18.9 e^{-3 s} / 21.0 s+1$, $P_{21}(s)=6.6 e^{-7 s} / 10.9 s+1$ and $P_{22}(s)=-19.4 e^{-3 s} / 14.4 s+1$. Then it is modeled in MATLAB SIMULINK as shown in Fig. 5, and running in real-time workshop to simulate the simplified dynamics of a distillation column. The virtual distillation column interacts with the outside through a multi-function analog and digital $\mathrm{I} / \mathrm{O}$ card (PCI-DAS1602-16) from Measurement Computing Corporation.

According to the disturbance decoupling control proposed in [17], two modified first order ADRC are designed to control $P_{11}(s)$ and $P_{22}(S)$ respectively. The control algorithm is coded and compiled in OpenPCS, an IEC (International Electrotechnical Commission) 61131-3 compatible PLC (Programmable Logic Controller) programming

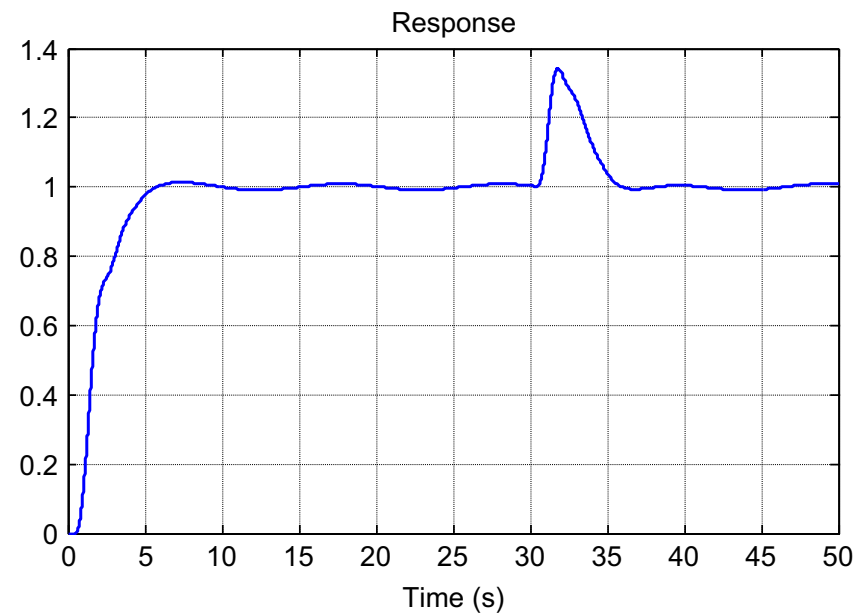

Fig. 4. Simulation results of nonlinear, time-varying system with time delay. environment as shown in Fig. 6, and then downloaded to and executed on the UPAC (Universal Programmable Automation Controller) platform as shown in Fig. 7, a product from UniControl Inc., which interfaces with the virtual distillation column.

The test is done with the following parameters: $\hat{b}_{1}=0.766$, $\omega_{c 1}=0.08 \mathrm{rad} / \mathrm{s}, \omega_{01}=0.8 \mathrm{rad} / \mathrm{s}, \hat{b}_{2}=-1.347, \omega_{c 2}=0.1 \mathrm{rad} / \mathrm{s}$ and $\omega_{02}=1 \mathrm{rad} / \mathrm{s}$. The set-points for loop 1 and loop 2 are $96 \%$ and $0.5 \%$ respectively. At $0 \mathrm{~s}$ an external disturbance with a magnitude of $0.2 \mathrm{lb} / \mathrm{min}$ is added to loop 1 , and another external disturbance with the same magnitude is added to loop 2 at $100 \mathrm{~s}$. The dynamics of the distillation column is simulated at a rate of $100 \mathrm{~Hz}$, and the controller runs at a rate of $10 \mathrm{~Hz}$. The test results are shown in Figs. 8 and 9 .

The figures show that the simulation and test results match well, and the disturbances are well rejected. In this experiment, the implementation of the proposed method on an industrial platform is demonstrated. Also its ability to deal with multi-inputmulti-output (MIMO) system with time delay, which is very common in real industrial applications, is verified. The tuning for such systems needs more consideration of the trade-off between the performances of each loop.

\section{Stability analysis}

The stability analysis of the proposed design for LTI time-delay systems is carried out by the following method presented in Ref. [32] by means of linear matrix inequality (LMI). Applying the modified ADRC design, the overall closed-loop system can be written as

$\dot{\mathbf{x}}(t)=\mathbf{A} \mathbf{x}(t)+\mathbf{A}_{\mathbf{d}} \mathbf{x}(t-\tau), t>0$

$\mathbf{x}(t)=\xi(t), \quad t \in[-\tau, 0]$

where $\xi(t)$ is a vector-valued continuous function of time and $\tau$ is the constant time delay. For the FOPTD system (7) there are

$\mathbf{x}=\left[\begin{array}{l}y \\ \hat{y} \\ \hat{f}\end{array}\right], \mathbf{A}=\left[\begin{array}{ccc}-a & -k_{1} b / \hat{b} & -b / \hat{b} \\ 0 & -l_{1} & 1 \\ 0 & -l_{2} & 0\end{array}\right]$ and $\mathbf{A}_{\mathbf{d}}=\left[\begin{array}{ccc}0 & 0 & 0 \\ l_{1} & -k_{1} & -1 \\ l_{2} & 0 & 0\end{array}\right]$

and for the SOPTD system (8) there are

$\mathbf{x}=\left[\begin{array}{c}y \\ \dot{y} \\ \hat{y} \\ \hat{y} \\ \hat{f}\end{array}\right], \mathbf{A}=\left[\begin{array}{ccccc}0 & 1 & 0 & 0 & 0 \\ -a_{0} & -a_{1} & -k_{1} b / \hat{b} & -k_{2} b / \hat{b} & -b / \hat{b} \\ 0 & 0 & -l_{1} & 1 & 0 \\ 0 & 0 & -l_{2} & 0 & 1 \\ 0 & 0 & -l_{3} & 0 & 0\end{array}\right]$ and

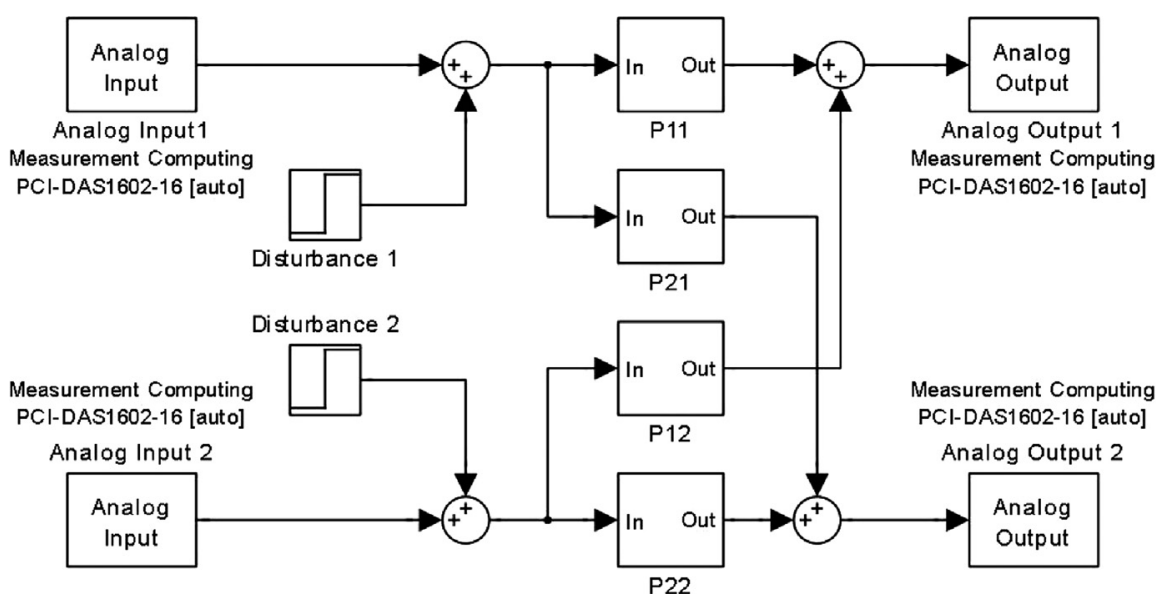

Fig. 5. SIMULINK model of the distillation column. 


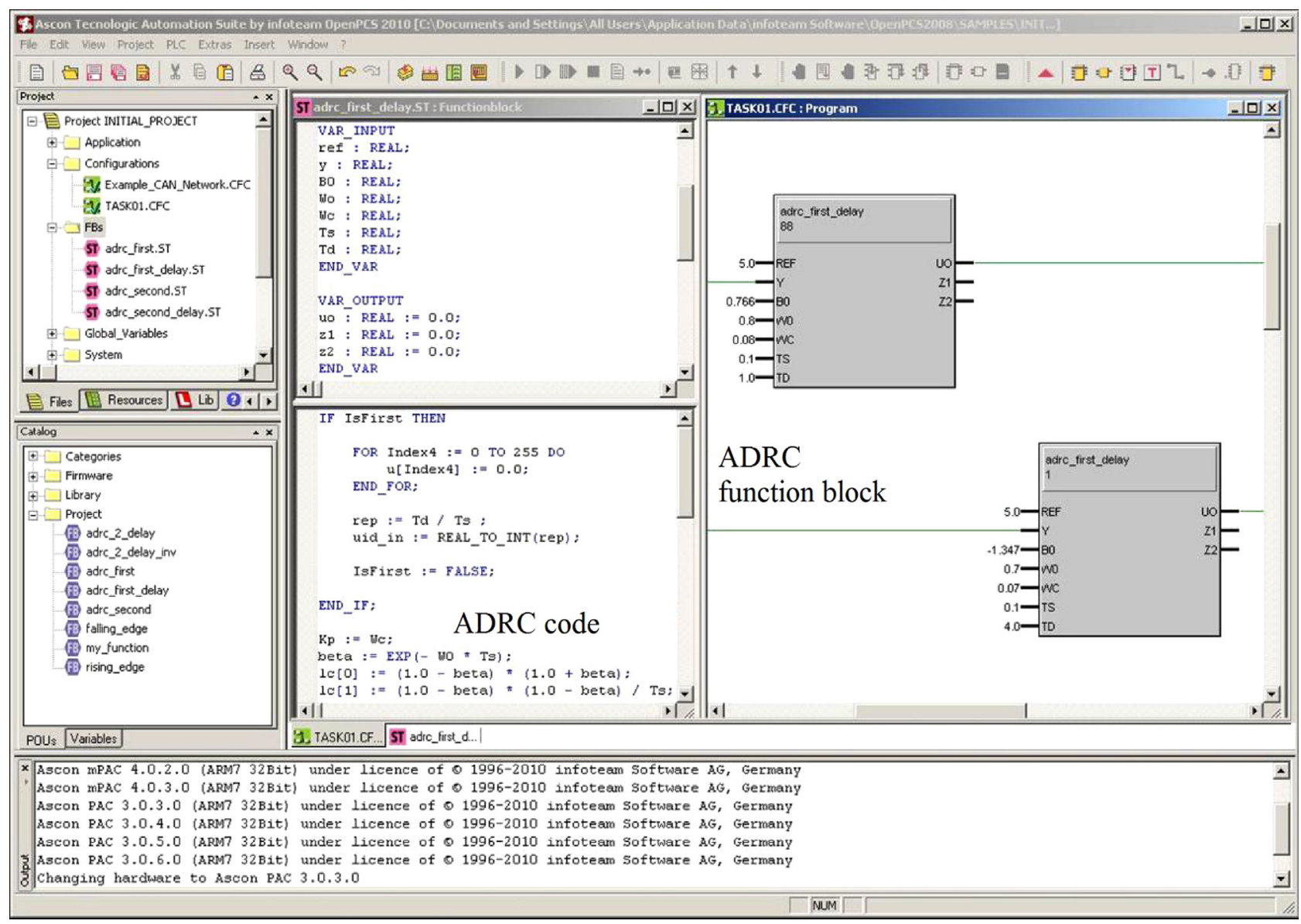

Fig. 6. OpenPCS programming environment.

$$
\mathbf{A}_{\mathbf{d}}=\left[\begin{array}{ccccc}
0 & 0 & 0 & 0 & 0 \\
0 & 0 & 0 & 0 & 0 \\
l_{1} & 0 & 0 & 0 & 0 \\
l_{2} & 0 & -k_{1} & -k_{2} & -1 \\
l_{3} & 0 & 0 & 0 & 0
\end{array}\right]
$$

The following lemmas will be used for the analysis.

Lemma 1. (Jensen's inequality) [33]: For any constant matrix $\mathbf{S}>0$, scalars $t_{2}>t_{1}$, vector function $\mathbf{x}:\left[t_{1}, t_{2}\right] \rightarrow R^{m}$ such that the integrations in the following are well defined, then $\left(t_{2}-t_{1}\right) \int_{t_{1}}^{t_{2}} \mathbf{x}(t)^{T}$ $\mathbf{S x}(t) d t \geq\left[\int_{t_{1}}^{t_{2}} \mathbf{x}(t) d t\right]^{T} \mathbf{S}\left[\int_{t_{1}}^{t_{2}} \mathbf{x}(t) d t\right]$.

Lemma 2. (Finsler's Lemma) [32]: Let $\mathbf{x} \in R^{n}, \mathbf{P}=\mathbf{P}^{T} \in R^{n \times n}$ and $\mathbf{H} \in R^{m \times n}$ such that $\operatorname{rank}(\mathbf{H})=r<n$. The following are equivalent:

1. $\mathbf{x}^{T} \mathbf{P x}<0, \quad \forall \mathbf{H} \mathbf{x}=0, \quad \mathbf{x} \neq 0$;

2. $\exists \mathbf{X} \in R^{n \times m}$ such that $\mathbf{P}+\mathbf{X H}+\mathbf{H}^{T} \mathbf{X}^{T}<0$.

Lemma 3. (Schur complements) [34]: For any symmetric matrix $\boldsymbol{\Psi}=\left[\begin{array}{cc}\mathbf{A} & \mathbf{B} \\ \mathbf{B}^{T} & \mathbf{C}\end{array}\right]$, where $\mathbf{C}>0$ and is invertible, then $\boldsymbol{\Psi}>0$ if and only if $\mathbf{A}-\mathbf{B C}^{-1} \mathbf{B}^{T}>0$.

Theorem 1. System (12) is asymptotically stable, if there exist symmetric matrices $\mathbf{P}>0, \mathbf{Q}>0$ and $\mathbf{M}>0$ such that the following LMI holds.

$$
\left[\begin{array}{ccc}
\mathbf{P A}+\mathbf{A}^{T} \mathbf{P}+\mathbf{Q}-\mathbf{M} & \mathbf{P A}_{\mathbf{d}}+\mathbf{M} & \tau \mathbf{A}^{T} \mathbf{M} \\
\mathbf{A}_{\mathbf{d}}^{T} \mathbf{P}+\mathbf{M} & -\mathbf{Q}-\mathbf{M} & \tau \mathbf{A}_{\mathbf{d}}^{T} \mathbf{M} \\
\tau \mathbf{M A} & \tau \mathbf{M} \mathbf{A}_{\mathbf{d}} & -\mathbf{M}
\end{array}\right]<0
$$

Proof. Choosing the following Lyapunov functional

$V(\mathbf{x}(t))=\mathbf{x}^{T}(t) \mathbf{P} \mathbf{x}(t)+\int_{t-\tau}^{t} \mathbf{x}^{T}(s) \mathbf{Q} \mathbf{x}(s) d s+\tau \int_{-\tau}^{0} \int_{t+s}^{t} \dot{\mathbf{x}}^{T}(\sigma) \mathbf{M} \dot{\mathbf{x}}(\sigma) d \sigma d s$

The time derivative of $V(\mathbf{x}(t))$ along the trajectory of system (12) is calculated as

$$
\begin{aligned}
\dot{V}(\mathbf{x}(t))= & 2 \mathbf{x}^{T}(t) \mathbf{P} \dot{\mathbf{x}}(t)+\mathbf{x}^{T}(t) \mathbf{Q} \mathbf{x}(t)-\mathbf{x}^{T}(t-\tau) \mathbf{Q} \mathbf{x}(t-\tau) \\
& +\tau^{2} \dot{\mathbf{x}}^{T}(t) \mathbf{M} \dot{\mathbf{x}}(t)-\tau \int_{t-\tau}^{t} \dot{\mathbf{x}}^{T}(\sigma) \mathbf{M} \dot{\mathbf{x}}(\sigma) d \sigma
\end{aligned}
$$

From Lemma 1, the following is true.

$-\tau \int_{t-\tau}^{t} \dot{\mathbf{x}}^{T}(\sigma) \mathbf{M} \dot{\mathbf{x}}(\sigma) d \sigma \leq-\tilde{\mathbf{x}}^{T}(t) \mathbf{M} \tilde{\mathbf{x}}(t)$

where $\tilde{\mathbf{x}}(t)=\mathbf{x}(t)-\mathbf{x}(t-\tau)$. Then

$$
\dot{V}(\mathbf{x}(t)) \leq \boldsymbol{\phi}^{T}(t) \boldsymbol{\Lambda} \phi(t)
$$

where $\boldsymbol{\phi}(t)=\left[\dot{\mathbf{x}}^{T}(t), \mathbf{x}^{T}(t), \mathbf{x}^{T}(t-\tau), \tilde{\mathbf{x}}^{T}(t)\right]^{T}$ and

$$
\boldsymbol{\Lambda}=\left[\begin{array}{cccc}
\tau^{2} \mathbf{M} & \mathbf{P} & 0 & 0 \\
\mathbf{P} & \mathbf{Q} & 0 & 0 \\
0 & 0 & -\mathbf{Q} & 0 \\
0 & 0 & 0 & -\mathbf{M}
\end{array}\right]
$$

According to Eq. (12), there is $\overline{\mathbf{A}} \boldsymbol{\phi}(t) \equiv 0$, where

$$
\overline{\mathbf{A}}=\left[\begin{array}{cccc}
\mathbf{I} & -\mathbf{A} & -\mathbf{A}_{\mathbf{d}} & 0 \\
0 & -\mathbf{I} & \mathbf{I} & \mathbf{I}
\end{array}\right]
$$




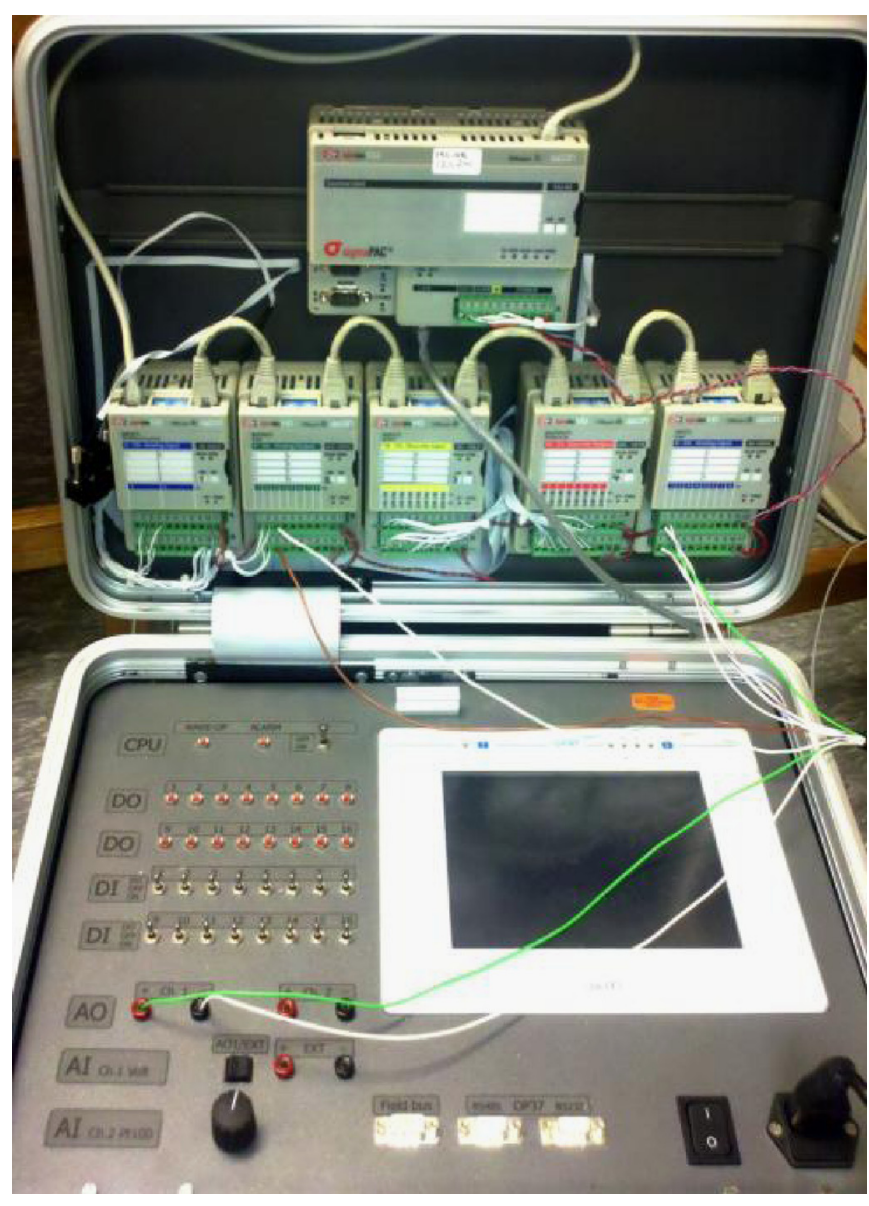

Fig. 7. UPAC platform.

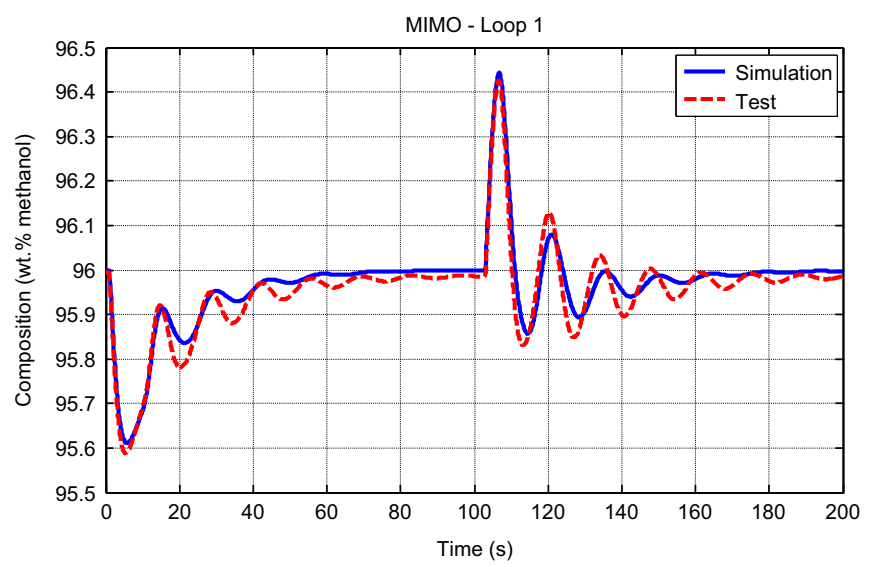

Fig. 8. Simulation and test results of loop 1 of the distillation column.

Then the closed-loop system (12) is asymptotically stable if for all $\overline{\mathbf{A}} \boldsymbol{\phi}(t)=0, \boldsymbol{\phi}^{T}(t) \boldsymbol{\Lambda} \phi(t)<0$.With

$\overline{\mathbf{A}}^{\perp}=\left[\begin{array}{cccc}\mathbf{A}^{T} & \mathbf{I} & 0 & \mathbf{I} \\ \mathbf{A}_{\mathbf{d}}^{T} & 0 & \mathbf{I} & -\mathbf{I}\end{array}\right]^{T}$,

which is orthogonal to $\overline{\mathbf{A}}$ and $\overline{\mathbf{A A}}^{\perp}=0$, from Lemma 2, the following holds.

$\boldsymbol{\phi}^{T}(t) \boldsymbol{\Lambda} \boldsymbol{\phi}(t)<0 \Leftrightarrow \overline{\mathbf{A}}^{\perp T} \boldsymbol{\Lambda} \overline{\mathbf{A}}^{\perp}<0$

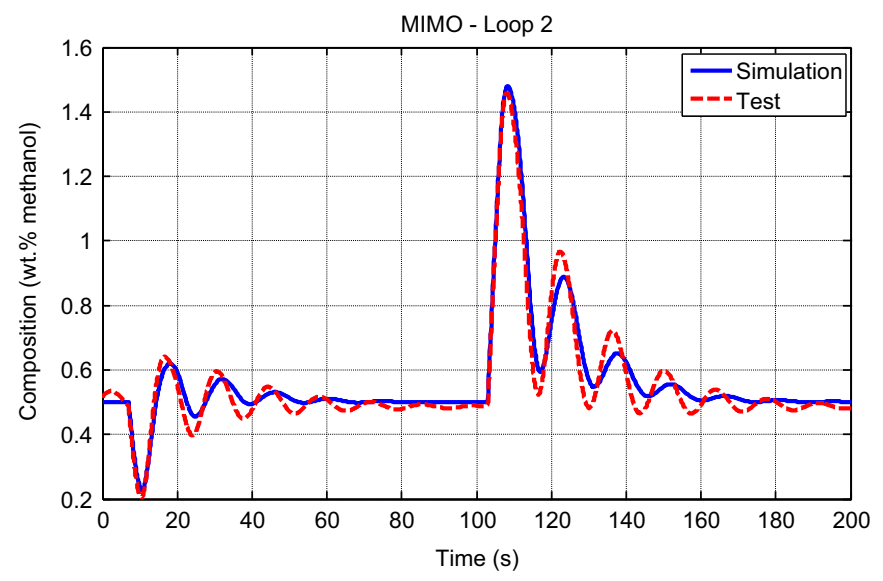

Fig. 9. Simulation and test results of loop 2 of the distillation column.

$\overline{\mathbf{A}}^{\perp T} \boldsymbol{\Lambda}^{\perp}=\left[\begin{array}{cc}\tau^{2} \mathbf{A}^{T} \mathbf{M} \mathbf{A}+\mathbf{P A}+\mathbf{A}^{T} \mathbf{P}+\mathbf{Q}-\mathbf{M} & \tau^{2} \mathbf{A}^{T} \mathbf{M} \mathbf{A}_{\mathbf{d}}+\mathbf{P} \mathbf{A}_{\mathbf{d}}+\mathbf{M} \\ \tau^{2} \mathbf{A}_{\mathbf{d}}{ }^{T} \mathbf{M} \mathbf{A}+\mathbf{A}_{\mathbf{d}}^{T} \mathbf{P}+\mathbf{M} & \tau^{2} \mathbf{A}_{\mathbf{d}}^{T} \mathbf{M} \mathbf{A}_{\mathbf{d}}-\mathbf{Q}-\mathbf{M}\end{array}\right]<0$

By Lemma 3, Eq. (19) is equivalent to Eq. (13). Thus, a sufficiently small $\varepsilon>0$ can always be found such that $\dot{V}(\mathbf{x}(t))<-\varepsilon\|\mathbf{x}(t)\|^{2}$, which ensures the asymptotic stability of system (12). Q.E.D.

Theorem 1 can be used as a guide for setting the controller parameters initially, since it guarantees the system stability. By fixing the ratio between the observer bandwidth and the controller bandwidth, a maximum controller bandwidth can then be found by solving the feasibility problem of Eq. (13). Since Eq. (13) is only a sufficient condition for stability, the solution may be conservative. Hence the controller bandwidth can be increased gradually until a satisfactory performance is achieved. For example, the ratio between the observer bandwidth and the controller bandwidth is chosen to be ten in Example 1, and the maximum controller bandwidth is found to be 0.004 , which is relatively close to the value 0.015 as used in the example.

\section{Conclusions}

In this paper, a modified ADRC design is proposed to control time-delay systems. It has been shown by the simulation that the synchronization modification effectively increases the achievable observer bandwidth of the ESO, which in turn improves the disturbance rejection performance over the regular ADRC design. Owing to the ability of ADRC to deal with internal uncertainties, the proposed design also turns out to be a unified solution for time-delay systems with stable, critical stable and unstable poles. The implementation of the proposed design on an industrial platform is done to demonstrate its practicality. The stability analysis of ADRC applied to time-delay systems is also done for the first time.

\section{Acknowledgments}

The authors want to thank UniControl Inc. for providing the UPAC platform based on which the experimental tests are conducted.

\section{References}

[1] Seborg DE, Edgar TF, Mellichamp DA. Process dynamics and control. 2nd ed. . Hoboken, NJ: Wiley; 2004. 
[2] Skogestad S, Postlethwaite I. Multivariable feedback control: analysis and design. Chichester, NY: Wiley; 1996.

[3] Smith OJM. A controller to overcome dead time. ISA Journal 1959;6:28-33.

[4] Normey-Rico JE, Camacho EF. Dead-time compensators: a survey. Control Engineering Practice 2008;16:407-28.

[5] Astrom KJ, Hang CC, Lim BC. A new Smith predictor for controlling a process with an integrator and long dead-time. IEEE Transactions on Automatic Control 1994;39:343-5.

[6] Matausek MR, Micic AD. A modified Smith predictor for controlling a process with an integrator and long dead-time. IEEE Transactions on Automatic Control 1996;41:1199-203.

[7] Normey-Rico JE, Camacho EF. Robust tuning of dead-time compensators for processes with an integrator and long dead-time. IEEE Transactions on Automatic Control 1999;44:1597-603.

[8] Stojic MR, Matijevic FS, Draganovic LS. A robust Smith predictor modified by internal models for integrating process with dead time. IEEE Transactions on Automatic Control 2001:46:1293-8.

[9] Zhong QC, Mirkin L. Control of integral processes with dead-time. 2. Quantitative analysis. IEE Proceedings: Control Theory and Applications 2002;149: 291-6.

[10] Zhong QC, Normey-Rico JE. Control of integral processes with dead-time. 1. Disturbance observer-based 2 DOF control scheme. IEE Proceedings: Control Theory and Applications 2002;149:285-90.

[11] Zhong QC. Control of integral processes with dead time.3. Deadbeat disturbance response. IEEE Transactions on Automatic Control 2003;48:153-9.

[12] Wang B, Rees D, Zhong QC. Control of integral processes with dead time. Part IV: various issues about PI controllers. IEE Proceedings: Control Theory and Applications 2006;153:302-6.

[13] Normey-Rico JE, Camacho EF. A unified approach to design dead-time compensators for stable and integrative processes with dead-time. IEEE Transactions on Automatic Control 2002;47:299-305.

[14] Normey-Rico JE, Camacho EF. Control of dead-time processes. London: Springer; 2007.

[15] Han J. From PID to active disturbance rejection control. IEEE Transactions on Industrial Electronics 2009;56:900-6.

[16] Sun B, Gao ZA. DSP-based active disturbance rejection control design for a 1-kW H-bridge DC-DC power converter. IEEE Transactions on Industrial Electronics 2005;52:1271-7.

[17] Zheng Q, Chen Z, Gao Z. A practical approach to disturbance decoupling control. Control Engineering Practice 2009;17:1016-25.

[18] Vincent J, Morris D, Usher N, Gao Z, Zhao S, Nicoletti A, et al. On active disturbance rejection based control design for superconducting RF cavities.
Nuclear Instruments and Methods in Physics Research Section A: Accelerators Spectrometers, Detectors and Associated Equipment 2011;643:11-6.

[19] Goforth FJ, Zheng Q Gao Z. A novel practical control approach for rate independent hysteretic systems. ISA Transactions 2012;51:477-84.

[20] Zhao S, Gao Z. An active disturbance rejection based approach to vibration suppression in two-inertia systems. Asian Journal of Control 2013;15:350-62.

[21] Freidovich LB, Khalil HK. Performance recovery of feedback-linearizationbased designs. IEEE Transactions on Automatic Control 2008:53:2324-34.

[22] Guo B, Zhao Z. On the convergence of an extended state observer for nonlinear systems with uncertainty. Systems and Control Letters 2011;60:420-30.

23] Huang Y, Xue W. Active disturbance rejection control: methodology and theoretical analysis. Journal of Systems Science and Mathematical Sciences 2011;31:1111-29 (in Chinese).

24] Zheng Q Gao LQ Gao Z. On validation of extended state observer through analysis and experimentation. Journal of Dynamic Systems, Measurement and Control 2012;134:024505-6.

25] Han J. Active disturbance rejection control technique: the technique for estimating and compensating the uncertainties. 1st ed. Beijing: National Defense Industry Press; 2008 (in Chinese)

[26] Xia Y, Shi P, Liu GP, Rees D, Han J. Active disturbance rejection control fo uncertain multivariable systems with time-delay. IET Control Theory and Applications 2007;1:75-81.

[27] Gao Z, 2003. Scaling and bandwidth-parameterization based controller tuning. In: Proceedings of the American Control Conference, IEEE. New York, pp. 4989-96.

28] Zhao S, Xue W, Gao Z. Achieving minimum settling time subject to undershoot constraint in systems with one or two real right half plane zeros. Journal of Dynamic Systems, Measurement and Control 2013;134 (034505-034505).

[29] O'Dwyer A. Handbook of PI and PID controller tuning rules. London, NJ: World Scientific; 2003

[30] Tan W, Fang F, Tian L, Fu C, Liu J. Linear control of a boiler-turbine unit: analysis and design. ISA Transactions 2008:47:189-97.

[31] Wood RK, Berry MW. Terminal composition control of a binary distillation column. Chemical Engineering Science 1973;28:1707-17.

[32] Xia Y, Fu M, Peng S. Analysis and Synthesis of Dynamical Systems with TimeDelays. Berlin, Heidelberg: Springer-Verlag; 2009.

[33] Gu K, Kharitonov V, Chen J. Stability of time-delay systems. Boston, MA Birkhäuser; 2003.

[34] Zhang F. The Schur complement and its applications. New York: Springer Science; 2005 